\title{
Multibiometric Personal Identification based on Hybrid Artificial Intelligence Technique using Serial Mode Architecture
}

\author{
Laheeb M. Alzoubiady, Ph.D \\ Dept. of Software Engineering \\ College of Computer Sciences \& Math. \\ University of Mosul ,Nenavha, IRAQ
}

\author{
Ibrahim A. Saleh, Ph.D \\ Dept. of Software Engineering \\ College of Computer Sciences \& Math. \\ University of Mosul ,Nenavha, IRAQ
}

\begin{abstract}
In this research a multibiometric identification system with serial mode using palmprint, Dental and DNA biometric traits is proposed to identify person. A proposed system consists of three steps, first step is to create a database where all the information about the authenticated users is stored. Second step is to extracts the features from a database images for palmprint and dental estimated the eigenvalues using KL transforming while the DNA data converted into ASCII code. The last step used hybrid Artificial technique combined backpropagation Neural Network and particle swarm optimization (PSO) as classifier and compare the test image with the stored information in the database. The experimental results applied cascaded (serial) mode show that the proposed system has an encouraging performance. The Genuine acceptance rate 98\%, False Acceptance Rate (2\%), False Rejected Rate (5\%) and Recognition Rate (93\%)
\end{abstract}

\section{Keywords}

Index Terms -Multibiometric - serial mode-

KL transform- backpropagation-Particle swarm intelligenceVerification- Identification

\section{Introduction}

Because of the rapid scientific development in the field of technology to authenticate persons to achieve high security in all spheres of our life, and to meet the increased security requirements in a variety of applications ranging from international border crossing to accessing personal information. It must used a biological traits (physical (iris, fingerprint, palm print, face, hand geometry, gait, voice. etc.) or behavioral (name, social security number, signature, etc...) ) to authenticate people. Used a biometric in personal identity verification are considered more reliable, especially if you use more than one traits, because these traits do not change over time[1][2].

Most of personal identity system use only a single biometric called mono-biometric, simply called biometric systems that cover only a single biometric trait are generally unable to provide the desired performance requirements as they suffer from problems like: noisy-data, spoof attacks, non-universality, insufficient population coverage, and improper sensor adjustment. To overcome these difficulties multi-biometric systems are used [3][4].

Using multi biometric traits to design a personal identity system capable of utilizing, more than one physiological or behavioral traits for identify person. It is generally believed that by integrating multi biometric traits into one single unit, the

limitations of mono-biometric systems can be alleviated. Given that several biometric sources usually compensate for the weaknesses of single biometric fusion techniques has dealt primarily with the fusion at the score matching leve [5 ].

The purpose of this paper is to create multi biometric personal identity system with serial mode integrating multiple traits using palmprint, dental and DNA traits to become system more reliable, These three traits sufficient to achieve the identity of the person at any time and different circumstances, either in postmortem (PM) and antemortem (AM), even if it became a long time on death. Also this system enable to universality, distinctiveness, uniqueness and permanence. The serial mode is more reliable from search in large database from different traits.

The rest of the paper is organized as follows: section 2 gives the background work from past researcher, Section 3 the overview of multibiometric system model and why used the serial mode. Section 4 fundemental of biometrics system. Section 5 suggest the overview of proposed technology, Section 6 provide the experimental result. Section 7 the conclusion the result.

\section{Background Work}

Many researches for person authentication using multi biometric concepts with different traits are done; table (1) summarized most important 
Table 1 Related work for person authentication using multi biometric concepts with different traits

\begin{tabular}{|c|c|c|c|}
\hline $\begin{array}{l}\text { Research } \\
\text { er }\end{array}$ & years & $\begin{array}{l}\text { Multibiometric } \\
\text { properties }\end{array}$ & Algorithm \\
\hline $\begin{array}{l}\text { Fierrez J. } \\
\text { A., et al. }\end{array}$ & 2003 & $\begin{array}{lr}\text { Fingerprint } & \text { and } \\
\text { an } & \text { online } \\
\text { signature } & \end{array}$ & $\begin{array}{l}\text { Applied HMM (Hidden } \\
\text { Markov Models) modeling } \\
\text { of temporal functions. The } \\
\text { classification by SVM } \\
\text { (Support Vector Machine) } \\
\text { [7]. }\end{array}$ \\
\hline $\begin{array}{l}\text { Toh K.A., } \\
\text { et al. }\end{array}$ & 2004 & $\begin{array}{l}\text { Fingerprint, hand } \\
\text { geometry and } \\
\text { voice }\end{array}$ & $\begin{array}{l}\text { Applied weighted-sum-rule } \\
\text { based match-score-level } \\
\text { fusion. In this research a } \\
\text { local decision can be } \\
\text { improved once threshold } \\
\text { settings are appropriately } \\
\text { selected for each user [8]. }\end{array}$ \\
\hline $\begin{array}{l}\text { Snelick R., } \\
\text { et al. }\end{array}$ & 2005 & $\begin{array}{l}\text { Three fingerprint } \\
\text { recognition } \\
\text { commercial } \\
\text { systems and one } \\
\text { face recognition } \\
\text { commercial } \\
\text { system }\end{array}$ & $\begin{array}{l}\text { Seven score normalization } \\
\text { techniques (min-max, z- } \\
\text { score, tanh, adaptive, two } \\
\text { quadrics, logistic, and } \\
\text { quadric-line-quadric) and } \\
\text { fusion techniques on the } \\
\text { normalized scores (simple } \\
\text { sum, min score, max score, } \\
\text { matcher weighting, and user } \\
\text { weighting)[9]. }\end{array}$ \\
\hline $\begin{array}{l}\text { Zhou X. ., } \\
\text { et al. }\end{array}$ & 2008 & $\begin{array}{l}\text { Face and video } \\
\text { gait }\end{array}$ & $\begin{array}{l}\text { Present a prediction model } \\
\text { that is based on the } \\
\text { likelihood ratio. They } \\
\text { derive the Fisher } \\
\text { measurement for the sensor } \\
\text { fusion system and matched } \\
\text { with score Gaussian } \\
\text { distributions [10]. }\end{array}$ \\
\hline Karthik N. & 2008 & $\begin{array}{l}\text { Fingerprint and } \\
\text { face }\end{array}$ & $\begin{array}{l}\text { Used fusion methodology } \\
\text { based on the Neyman- } \\
\text { Pearson theorem for } \\
\text { combination of match scores } \\
\text { provided by multiple } \\
\text { biometric matchers [11]. }\end{array}$ \\
\hline Park U. & 2009 & $\begin{array}{l}\text { Video-bases face } \\
\text { recognition using } \\
\text { framework using } \\
\text { 3D face modeling } \\
\text { technique }\end{array}$ & $\begin{array}{l}\text { Used fusion scheme that } \\
\text { combines the facial mark } \\
\text { matching with a commercial } \\
\text { face recognition matcher } \\
\text { with eclidlan distance to } \\
\text { improve the recognition } \\
\text { performance [12]. }\end{array}$ \\
\hline $\begin{array}{l}\text { Marasco } \\
\text { E. }\end{array}$ & 2010 & $\begin{array}{l}\text { Multimodal } \\
\text { biometrics system } \\
\text { to be protected } \\
\text { against number of } \\
\text { vulnerable }\end{array}$ & $\begin{array}{l}\text { In proposed points that } \\
\text { may be attacked by a hacker } \\
\text { who may choose to fake } \\
\text { only a subset of them to } \\
\text { improve the performance of } \\
\text { the existing integration } \\
\text { mechanisms in presence of } \\
\text { degraded data and their } \\
\text { security in presence of } \\
\text { spoof attacks [5]. }\end{array}$ \\
\hline Richard Y. & 2011 & $\begin{array}{l}\text { Face and iris } \\
\text { images }\end{array}$ & $\begin{array}{l}\text { Applied algorithms can be } \\
\text { used to detect the subject's } \\
\text { face, locate the eyes, reduce } \\
\text { iris noise, segment the iris, } \\
\text { generate a template and then } \\
\text { identify the subject through } \\
\text { typical pattern matching } \\
\text { algorithms[13]. }\end{array}$ \\
\hline $\begin{array}{l}\text { Al-Mayyan } \\
\text { W. }\end{array}$ & 2012 & Signature and iris & $\begin{array}{l}\text { Wwhich combined the } \\
\text { feature-level and decision- } \\
\text { level fusions, have improved } \\
\text { the final authentication } \\
\text { performance [14]. }\end{array}$ \\
\hline
\end{tabular}

\section{Multibiometric System}

Multibiometric system is involved one of three modes (serial, parallel or hierarchical) [15]. Serial mode, sometimes called cascade mode is used in this research. Serial mode uses more than one biometric trait. If the output of one matching operation on one biometric source is sufficient to authenticate the user, no other biometric samples are processed further.

In serial mode a decision could be made before acquiring all the samples. Therefore, multiple biometric traits do not have to be captured at the same time. Furthermore, a decision could be obtained before acquiring the rest of traits. As a result, the overall recognition duration can be decreased[16]. For example, in proposed authentication system based on palmprint, dental and DNA (see figure 1). Initially the user uses the palmprint validation unit, and if this fails dental validation is applied. If the last validation is failed the DNA unit is required. The reward of such systems is that many users will enroll to the system using single trait.

The cascading scheme can improve the user convenience as well as allow fast and efficient searches in large scale identification tasks. For example, when a cascaded multimodal biometric system has sufficient confidence on the identity of the user after processing the first modality, the user may not be required to provide the other modalities. The system can also allow the user to decide which modality he/she would present first [17].

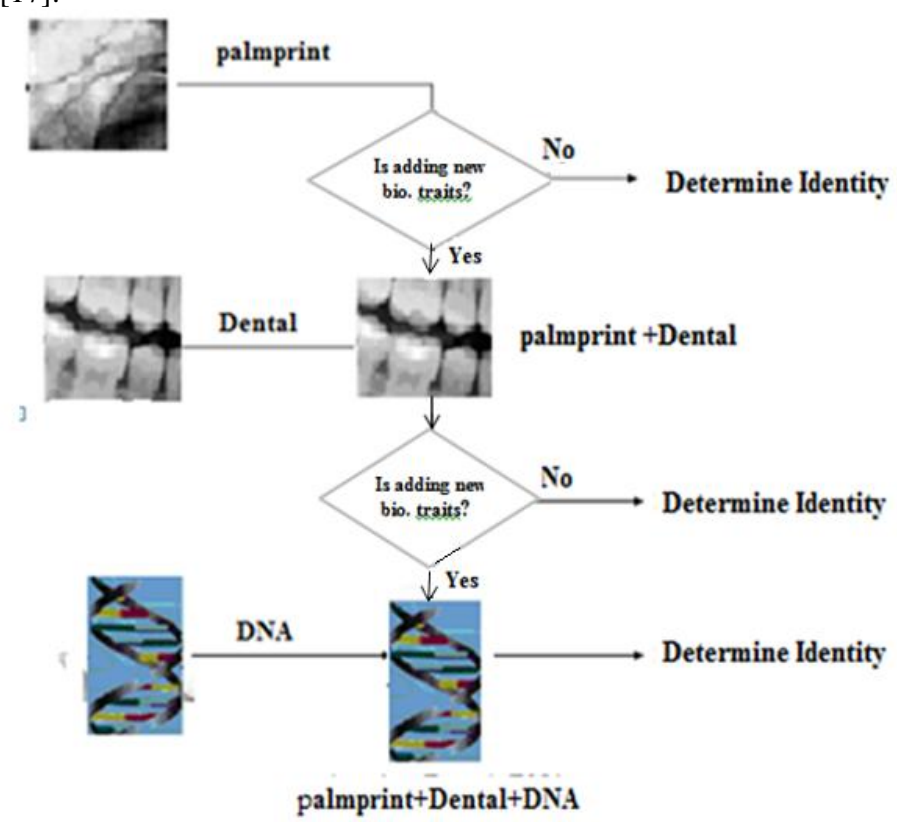

Figure (2): Serial mode multibiometric

When the system is faced with the task of identifying the user from a large database, it can utilize the outcome of each modality to successively prune the database, thereby making the search faster and more efficient. Thus, a cascaded system can be more convenient to the user and generally requires less recognition time when compared to its parallel counterpart. However, it requires robust algorithms to handle the different sequence of events $[17][18]$.

\section{Biometrics System Fundamentals}

Biometric systems can be categorized into three main functionalities as shown in figure (2), enrollment, verification and Identification [19]. During Enrollment, as shown in figure (2a), a sample of the biometric trait is captured, processed by a 
computer, and stored for later comparison the flow of information in verification and identification".

In verification function, also or sometime it is called "authentication", see figure (2b) the user claims an identity and the system verifies whether the user claims an identity and the system verifies whether the claim is genuine, i. e., the system answers the question, the query is compared only to the template corresponding to the claimed identity (a one-to-one match)[20]. If the user's input and the template of the claimed identity have a high degree of similarity, then the claim is accepted as "genuine". Otherwise, the claim is rejected and the user is considered an "impostor". In the biometric literature, the terms "client" or "authentic" are sometimes used in place of the term "genuine" [21].

(a) Enrollment

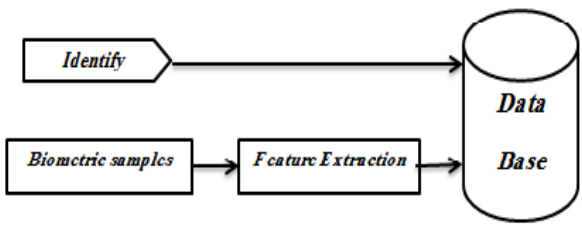

(b) Verification

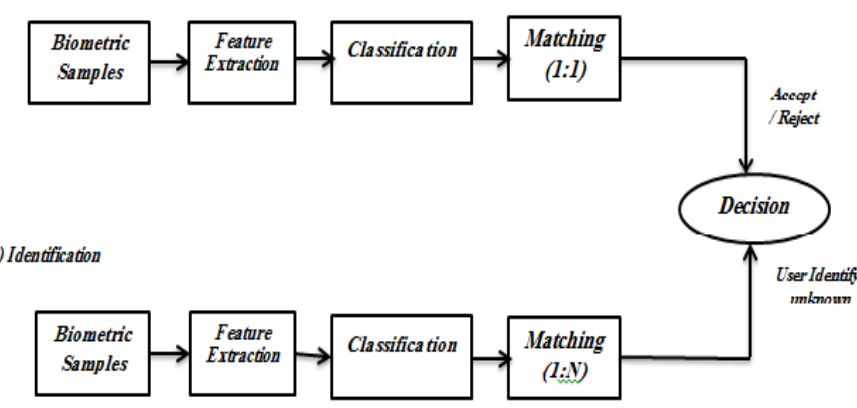

Figure (2): Biometric Functionalities (a) Enrollment (b) verification (c) Identification [20]

Identification function, see figure $(2 \mathrm{c})$ refers where a person's identity must be initially established. Acquired data associated with any person that identified is compared with stored template with database. It depending the compression (one-tomany), whose templates highest degree of similarity between the user's inputs and the outputs by the system. Typically, if the highest similarity between the input and all the templates is less than a fixed minimum threshold, the system outputs a reject decision which implies that the user presenting the input is not one among the enrolled users [20].

\section{Proposed Multibiometric Personal Identification System Using Serial Mode Architecture}

This section explains design and implementation for the proposed multibiometric Personal Identification System and evaluation performance. It provides a brief overview of palmprint, dental and DNA traits to identification and verification any person, see figure (3)

To create a multibiometric Personal Identification System using palmprint, dental and DNA traits follow these steps:

Step 1:.Data Captured: first step is to captured information for palmprint, dental, and DNA used as input to the system .To captured the palmprint capturing device, setting the mode 300 dot/inch due to make the process faster. A prototype for image acquiescing is shown in figure 4. Acquiesced image is then reduced to $25 \%$ both horizontally and vertically to make the image size small. It is necessary that the hand should be placed in a relaxed state and the fingers are separated from each other's to obtain very clear features of palm shown in figure(4).
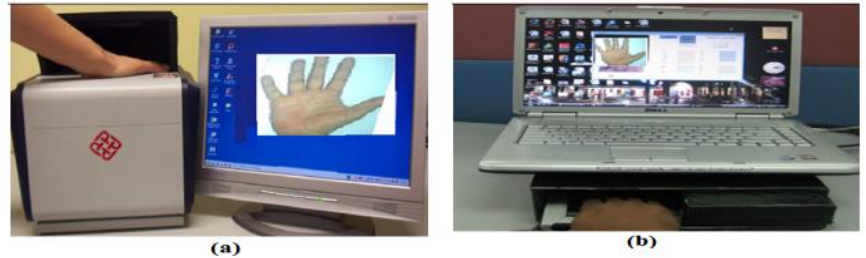

Figure (4): Prototype of the proposed multispectral palmprint system.

Palm is the inner surface of hand from wrist to the root of fingers. A print impression made in or on a surface by pressure. A palmprint is defined as the skin patterns of palm, composed of the physical characteristics of the skin patterns such as lines, wrinkles, points, ridges, valleys and texture [23].

For dental images are captured from dental radiographs called panoramic. A panoramic dental radiograph is a large, single $\mathrm{x}$ ray film that shows the bony structure of the dental and face. It is entirely extra oral, which means that the film remains outside of the mouth while the machine shoots the beam. The panoramic dental radiograph is a lower resolution image than intraoral film.

The DNA can be obtained from special device. In this research, DNA database are taken from National Science and Technology Council (NSTC) , Committee on Technology consist of fifty person each one has 200 samples for example Deoxyribonucleic acid (DNA) is the carrier of genetic material found in most organisms, including humans. Each individual human is identifiable by hereditary traits found in their DNA, which are located in the nucleus of the cells well as the mitochondria. DNA serves as a genetic code that is unique to every organism, no two being exactly alike. A single strand of DNA is a string consisting of a combination of four different base nucleotides shown in Figure (5):

1. Adenine: Its chemical form denoted by letter (A).

2. Guanine: Its chemical form denoted by letter $(\mathrm{G})$.

3. Cytosine: Its chemical form denoted by letter (C).

4. Thymine: Its chemical form denoted by letter (T) [24].

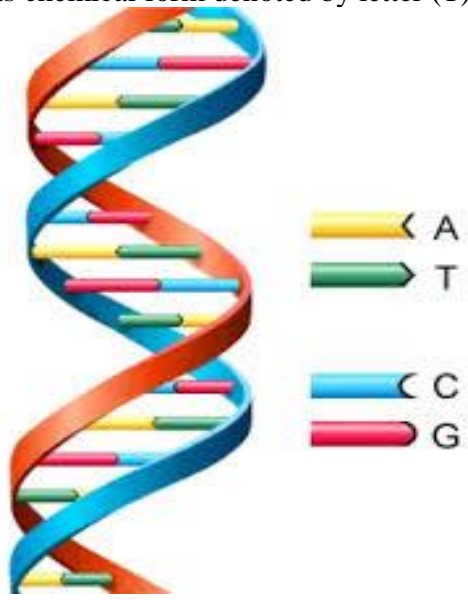

Figure (5) : DNA is double helix structure[25]

Step 2: Data Preprocessing: The second step is preprocessing the image captured in step 1, for palmprint captured image Readed as a color image, normalize it into fixed size $(256 * 256)$ pixcel and then convert colored 
image of palmprint into the gray scale image according to equation (1), see Figure (6).

$$
\text { Gray }=0.2989 X * R+0.7870 X * G+0.1140 X * B \text {. }
$$

Where R: represent red color, G: represent green color, B: represent blue color.

After that enhancement the image by remove noise from images with enhances filter called median filter matrix default 3-by-3 neighborhood, the median filter replaces all the image pixels in the same time with the median of the image pixel values in a predefined neighborhood of the given pixel. More precisely, if $f(x, y)$ denotes the pixel value in the position $(x, y)$ then the new $f^{\prime}(x, y)$ value of that position will be reckoned by Image Enhancement using Median Filters in algebraic reconstruction methods illustrate in equation 2):

$$
f^{\prime}(x, y)=\operatorname{med}_{-k \leq u, v \leq k}\{f(x+u, y+v)\}
$$

Where the median calculated over $(2 \mathrm{k}+1) *(2 \mathrm{k}+1)$ window, the size of filter has same value and $(\mathrm{u}, \mathrm{v})$ represents pixel position in the filter [120]. The result of enhancement has shown Figure (6).

After the image-enhanced step, the images are traced to obtain the contours of hand shape by rotate the hand image through angle ALPHA counter clockwise direction around an axis described by the one-element direction vector , coordinates to be more suitable for cropping that and illustrated in the Figure (6)

The next thing done is to create a bitmap of the image by cropping means removing unnecessary borders of hand image. This is done by cloning the useful part from original image taking a rectangle of the original as shown in Figure (6).

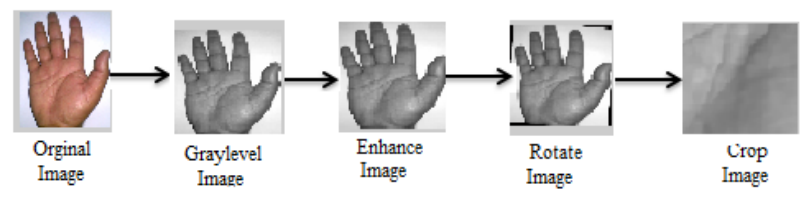

Figure (6): palmprint preprocessing stages

The dental normalization stages same as stages in palmprint as shown in figure (7), at first normalized the images into fixed size $\left(256^{*} 128\right)$ pixcel and then convert colored image of dental into the gray scale image, enhanced images (3-by-3) low pass filter called order filter, because this filter increase the sharpness and the contrast of spatially ordered detail patterns [27], and crop cropping part must consist of lateral incisors and central incisors.

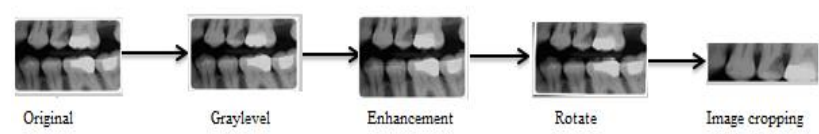

Figure (7): Dental preprocessing stages

In DNA preprocessing there is no noise to remove, or rotation except converting the letters in to their equivalent ASCII code numbers to become more suitable for input to the to the classification step.

Step 3 :Feature Extraction: Feature extraction refers to the process of generating a compact but expressive digital representation of the underlying biometric trait, called a template which contains the unique data to glean only the salient information from the acquired biometric sample to form a new representation of the biometric trait, called the feature set. The template is expected to contain only the salient discriminatory information that is essential for recognizing the person. To extract feature for palmprint and dental traits KL transform also known as the principal component transformation. Eigen value method is used set of orthonormal basis vectors. Each eigenvalue is derived from the covariance analysis of the image population. A similarity measure is then created in order to measure resemblance between images. Two images are considered to be similar if the distance between their feature vectors is small in the eigenvector feature space [28].

In Mathematic form a two dimension image can be represented as one dimension vector by concatenating each row (or column) into a long thin vector. The covariance matrix of the input data is calculated starting from the algorithmic mean of all vectors $\mathrm{I}_{1}, \mathrm{I}_{2}, \ldots \mathrm{I}_{\mathrm{i}}$.

$$
\psi=\frac{1}{M} \sum_{i=0}^{M} I_{i}
$$

The difference image vector Ii and mean is called with

$$
\Phi_{i}=I_{i}-\psi \text {. }
$$

The theoretical Covariance matrix $\mathrm{C}$ of all $\mathrm{i}$ is

$$
C=\frac{1}{M} \sum_{i=0}^{M} \Phi_{i} \Phi_{i}^{T}
$$

All eigenvectors $\mathrm{v}_{\mathrm{i}}$ and eigenvalues $\lambda_{\mathrm{i}}$ of this covariance matrix are derived from the relationship.

$$
\lambda_{i}=\frac{1}{M} \sum_{i=0}^{M}\left(v_{i}^{T} \Phi_{i}^{T}\right)^{2}
$$

The collection of $\mathrm{M}$ eigenvectors $\mathrm{V}_{\mathrm{i}}$ can be seen as the reduced dimension representation of the original input image. This set of eigenvectors will have a corresponding eigenvalues associated with it, which indicates the distribution of this eigenvector in representing whole dataset. The small set of eigenvalues is enough to build up the whole image characteristic.

$$
\varepsilon=\sum_{i=0}^{P} v_{i} \ldots \ldots .(7)
$$

Where $\mathrm{P}$ is number of eigenvalues.

KL transform is applied to images (palm and dental). This transformation reduce the original dimension of the image, thus the dimensions are greatly reduced and the most representative features of the whole dataset still remain within only eign features, see figure (8). While in DNA no feature extraction is used because there is no image except convert the serial number into their ASCII code.
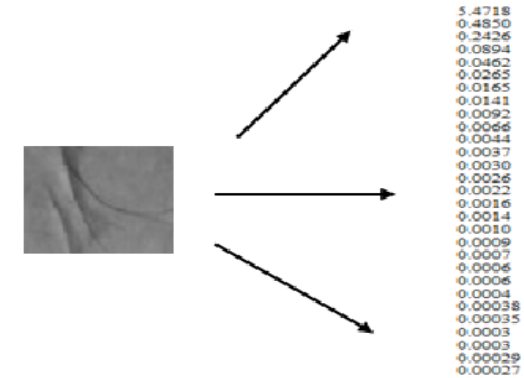

Figure (8): The eigenvalues extract from palmprint image.

Step5: Classification: To classify images first clustered the featured that obtain from KL transform algorithm by Particle swarm optimization (PSO) algorithm, because it has been largely applied to solve optimization problems. 
The standard approach is composed by a swarm of particles, where each one has a position within the search space and each position represents a solution for the problem, and then apply backpropagation artificial neural network is used as a matching method for palmprint and dental biometrics approach.

In a PSO system, a swarm of individuals (called particles) fly through the search space. Each particle represents a candidate solution to the optimization problem. In PSO each particle tries to modify its position using the following information [30].

$>$ The current positions $\left(\mathbf{x}_{\mathrm{i}}\right)$,

$>$ The current velocities (v),

$>$ The distance between the current position and $\mathrm{p}^{\text {best }}$,

$>$ The distance between the current position and the $\mathrm{g}^{\text {best }}$.

$>$ The personal best position of the particle $\left(\mathbf{y}_{\mathrm{i}}\right)$.

$>$ The neighborhood best position $\left(\hat{y}_{\mathbf{i}}\right)$.

Bellow represent PSO algorithm for clustering palm, dental and DNA traits in proposed system:

Step1: At initial stage, each particle randomly chooses $p$ different vectors from the document collection as the initial cluster centroid vectors,

- Population size 50.

- Max number of iteration $=100$

- Velocity limitation $\left(\mathrm{V}_{\max }\right)=8$.

- Inertia weight $\left(\mathrm{W}_{\max }\right)=0.9$.

- Inertia weight $\left(\mathrm{W}_{\min }\right)=0.5$.

- Acceleration constant (C1 and C2) 1.2

Step2: For each particle:

(a) Assign each document vector in the document set to the closest centroid vector.

(b) Calculate the fitness value based on equation (8).

$$
f=\frac{\sum_{i=1}^{N C}\left\{\frac{\sum_{j=1}^{p_{i}} d\left(o_{i}, m_{i j}\right)}{p_{i}}\right\}}{N_{c}}
$$

Where:

$\mathrm{m}_{\mathrm{ij}}$ : denotes the $\mathrm{j}_{\mathrm{th}}$ vector, which belongs to cluster $\mathrm{i}$;

$\mathrm{O}_{\mathrm{i}}$ : is the centroid vector of the $\mathrm{i}_{\mathrm{th}}$ cluster;

$\mathrm{d}\left(\mathrm{o}_{\mathrm{i}}, \mathrm{m}_{\mathrm{ij}}\right)$ : distance between document $\mathrm{m}_{\mathrm{ij}}$ and the cluster centroid $\mathrm{O}_{\mathrm{i}}$;

$\mathrm{p}_{\mathrm{i}}$ : stands for the number of documents, which belongs to cluster $\mathrm{C}_{\mathrm{i}}$;

and $\mathbf{N}_{\mathbf{c}}$ stands for the number of clusters.

(c) Using the velocity and particle position to update based on the $\mathrm{p}^{\text {best }}$ and $\mathrm{g}^{\text {best }}$ equations 9 and 10 and to generate the next solutions in equation( $(9)$.

$v_{i}(t+1)=v_{i}(t)+c_{1} * \operatorname{rand}() *\left(p_{i}(t)-x_{i}(t)\right)+c_{2} * \operatorname{rand}()\left(g_{i}(t)-x_{i}(t)\right) \ldots \ldots .(9)$

$p_{i}(t+1)=p_{i}+v_{i}(t+1) \quad$ for $i<i \leq N$

Where the $i$ value $(1<\mathrm{i}<\mathrm{N}), c_{1}$ and $c_{2}$ are constants known as acceleration coefficients and rand () are two separately generated uniformly distributed random numbers range $[0,1]$.

Step3: Repeat step (2) until one of the following termination conditions is satisfied.

(a) The maximum number of iterations is exceeded or

(b) The average change in centroid vectors is less than a predefined value [30].

Backpropagation BP is chosen among various other learning algorithms of ANN because it is linearity and power mapping of network. BP neural network algorithm has a parallel distributed processing multilayer architecture, called neurons.
Each neuron connected between them by weights [31]. The network consists of input nodes (x), hidden nodes $(\mathrm{z})$ and output nodes (y). Learning in neural network takes place by changing the weights The activation function uses the sigmoidal function as given below[31]:

$$
y_{j}=\frac{1}{1+e^{-x j}}
$$

The architecture of backpropagation ANN used in this research comprises by experience found the best values for neural network are the following: (see figure 9).

- Multilayer fully connected BP network (3 layers).

- There are 400 image represent 50 persons for identification approach 50 persons (8 image to each person) and 200 images for verification approach represent 50 persons (4 images to each person).

- 30 input nodes represent eigenvalues for each image.

- 10 hidden nodes.

- Learning Rate, $\eta=0.125$.

- Epsilon rate $=0.004$.

- The outputs is represented by 6 node (represent 50 person in binary code)

For getting faster performance, multilayer fully connected BP network is considered. Based on the 30 features the output will be generated either valid or invalid user therefore 30 inputs and one output nodes are preferred. In the experimental analysis, it has been observed that network's parameters are performed smoothly. Before verification, we need to train our neural network. Training phase begins only when the user doesn't provide any ID. The system treats him as a newcomer. Eight hand images are collected from each user.

Feature values are extracted from the hand images. Extracted feature values are used to train a Neural Network until error falls below the threshold. Back-propagation match pattern will generate (1) from the single output node, otherwise output will be varied. After the training is over, error and optimal weight vectors of the network will be stored with a newly assigned unique personal identity (ID). The person is informed about this ID which is used later by him for his verification.

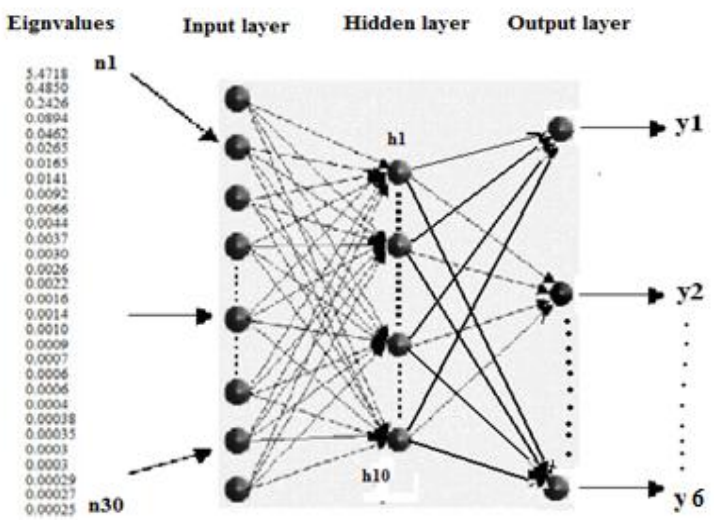

Figure(9): backpropagation neural network

To recognized any person depending on DNA biometrics characteristic, a sequence of four basis DNA is used as input to backpropagation artificial neural network from ( 200 input nodes, 30 hidden nodes, 6 output nodes for 50 persons. 
Classification is to be more suitable for input to the matching stage in identification or verification approach.

\section{Verification}

Verification system consists of two classes recognition assignment, where the sample is classified either genuine or not. The result with predefined allies between two values for threshold (minimum, maximum threshold) and classifies the claimer. The system accepts the claimer if and only if the value less than or equal the maximum threshold and greater than minimum threshold. ID is validated and matched with one of the images stored in the database.

Let $D_{\text {palmprin }} t, D_{\text {dental }}$ and $D_{\text {DNA }}$ denote the decision of the palmprint, dental and DNA respectively. The serial mode (shown in figure 9) can be obtained after training each classifier independency to take the final decision of the individual classifiers in order to find $\mathrm{D}_{\text {palmprint }}, \mathrm{D}_{\text {dental }}$ and $\mathrm{D}_{\mathrm{DNA}}$ the estimated by equation (12)

$$
D=\left(D_{\text {palmprint }} / / D_{\text {teeth }} / / D_{D N A}\right)
$$

Where // mean if failure rule.

The summarized of the algorithm applied in this research

Step 1: captured all the images samples.

Step2: Normalize the images to become more suitable for processing, and crop these images to remove unused part of image.

Step 3: Apply the KL transformation on the extract images to obtain eigenvalue and Eigen vector

Step 4: Extract the global features from palmprint and dental to obtain eigenvalues, while DNA is convert into ASCII code.

Step 5: Apply the eigenvalues those obtain in step 2 (except DNA) as input to PSO algorithm and then to the backpropagation supervisor neural network.

Step 6: Train set of identification images the network to 30-90 epochs with epsilon equal 0.001 and save the output for network.

Step 7 verify the image choosing any ID number belong any person in database set and train it in the same network.

Step 8: compare the output for matching with dataset of step five, and then decide if accepted or rejected

Step 9: if palm print rejected in decision in step 7, then apply the dental decision otherwise break the system for successor acceptable.

Step 10: if dental rejects the decision, then apply DNA decision otherwise break the system for successor acceptable

Step 11: if DNA is accepted then the system is accepted otherwise the system rejected.

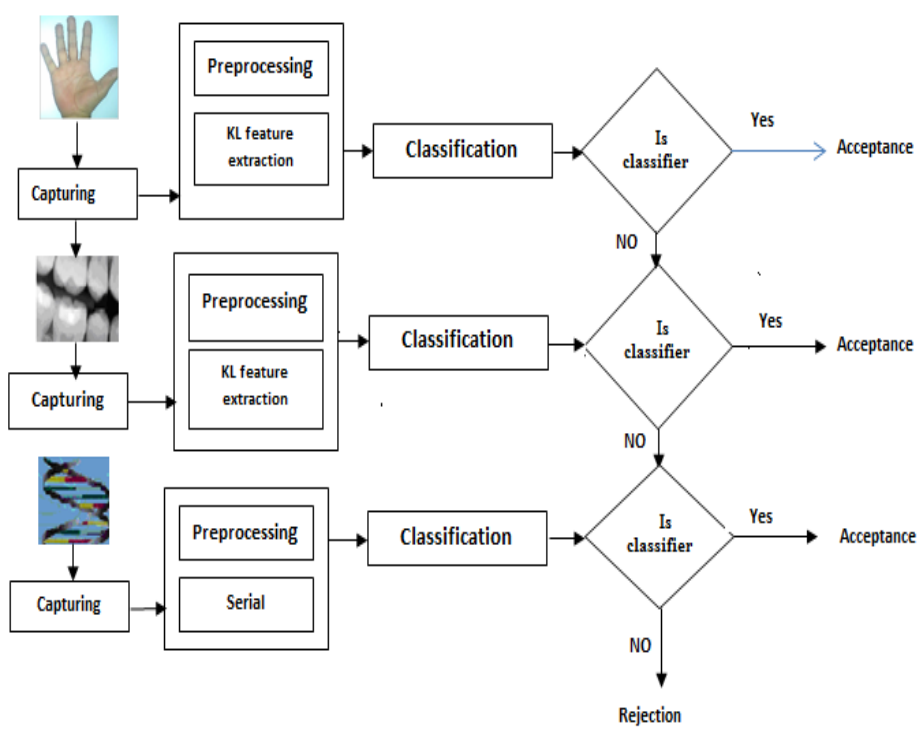

Figure (9): The proposed multibiometric system with serial mode architecture

\section{Experiment Evaluation}

In this research, a novel approach is presented to authenticate individuals by using serial multibiometric mode using palmprint, dental and DNA features. The virtual database collected from 50 different persons(eight images for each person). The hand images are captured from prototype device. The dental image captured from radiographs called panoramic (50 persons, four images for each person), while DNA captured with special device(50 person). Then do normalization images to appear suitable and comfortable for all users. The feature extraction using KL transform. Besides, we propose verification mechanisms in PSO and BP neural network-based method, to verify the system .The performance evaluation of the serial mode system four wellknown measurements are used, Genuine acceptance rate (GAR), False Rejection Rate (FRR), False Acceptance Rate (FAR), and recognition rate $(\mathrm{RR})$ of our proposed

- Genuine acceptance rate (GAR)(98\%)

- False Acceptance Rate(FAR) $(2 \%)$

- $\quad$ False Rejected Rate (FRR)(5\%)

- Recognition Rate (RR)(93\%).

Figure (10) represented the histogram from the performance evaluation from system

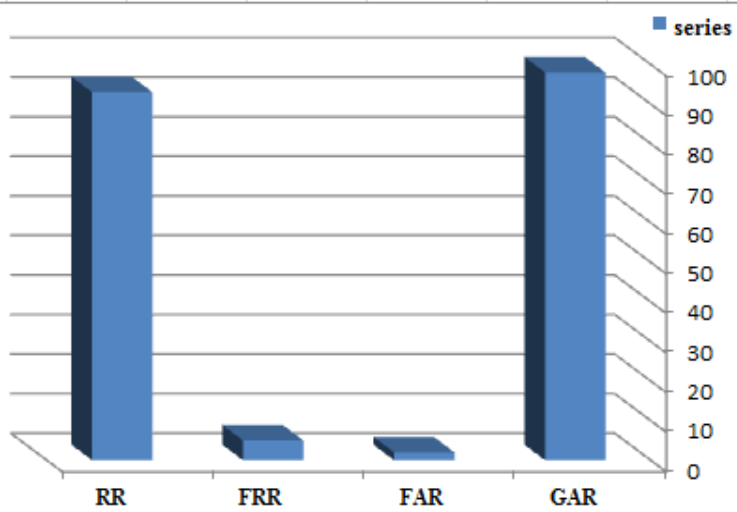

Figure (10) Histogram of serial multibiometric system 
Therefore automated multibiometric system designed to identify individuals from such pieces of multiple evidences, In this research from multibiometric can effectively achieve higher performance.

\section{Conclusion}

The essential objective of this research is to examine the performance of a multibiometric system can be improved by integrating complementary information which comes primarily from three different and independent modalities ( Palmprint, Dental and DNA). This Multibiometric system uses the three traits in serial mode is a good system since it enables to capture traits for one of those traits or when there is noise. It has been demonstrated that this method is efficient due to its high success rate. It is simple since by taking when large database and if there is one image from the user we can get the features for all the unimodal biometric employed.

Using particle swarm intelligence and PB neural network called (hybrid technique) is the best solution and more effective for multibiometric systems.

In future, an attempt will be made to develop a person identification system by using multibiometric technique with parallel architecture.

\section{References}

[1] Rashmi S. \&Payal J., 2012, "Multi Biometric System: Secure Security System "IJREAS Volume 2, Issue 2 ISSN: 22493905 International Journal of Research in Engineering \& Applied Sciences 182 http://www.euroasiapub.org

[2] National Biometric Security Project, 2008, "Biometric Technology Application Manual" Volume One: Biometric Basics, Updated Summer.

[3] Singh H. \& Gayathri R.,2012, " Image Authentication Technique Using Fsim Algorithm" International Journal of Engineering Research and Applications (IJERA) ISSN: 2248-9622 Vol. 2, Issue 2, pp.1129-1133. www.ijera.com

[4] Farhat Anwar, et al. 2008, "Multibiometric Systems Based Verification Technique" Faculty of Engineering, Department of ECE International Islamic, University Malaysia

[5] Marasco E. ,2010 "Secure Multbiometric Systems" PH.D thesis submitted to University of Naples Federico

[6] Chen A.K. Jain., 2005, "Dental biometrics: alignment and matching of dental radiographs. Pattern Analysis and Machine Intelligence", IEEE Transactions on, 27(8):13191326

[7] Fierrez J. -Aguilar, et al, 2003 'A comparative evaluation of fusion strategies for multimodal biometric verification", Proceedings of the 4th International Conference AudioVideo-Based Biometric Person Authentication, Vol. LNCS 2688, pp. 830-837.

[8] Toh K.A. et al . 2004 "Exploiting global and local decisions for multi-modal biometrics verification" IEEE Transactions on Signal Processing, Vol.52(10), pp. 3059-3072.

[9] Snelick R \&el al. 2005" Large Scale Evaluation of Multimodal Biometric Authentication Using State-of-the-Art Systems", IEEE Trans. Pattern Analysis and Machine Intelligence, Vol. 27(3), pp. 450-455,

[10] Zhou, X., and Bhanu B., 2008, "Feature fusion of side face and gait for video-based human identification' Pattern Recognition, 41(3), 778-795..

[11] Karthik K., 2008 , "Multibiometric Systems: Fusion Strategies and Template Security" Mc.S thesis Submitted to Michigan State University, Department of Computer Science and Engineering.

[12] Park U., 2009, "Face Recognition: face in video, age invariance, and facial marks" PH.D thesis submitted to Michigan State University, computer science.
[13] Richard Y. ,2011,“ Algorithm to Process and Measure Biometric Information Content in Low Quality Face and Iris Image" Ph.D thesis Faculty of Graduate and Postdoctoral Studies University of Ottawa Ottawa, Canada.

[14] Almayyan W. ,2012 "Performance Analysis of Multimodal Biometric Fusion " PhD Thesis Faculty of Technology De Montfort University England, United Kingdom February,

[15] Farhat A. , 2008, "Multibiometric Systems Based Verification Technique" Faculty of Engineering, Department of ECE International Islamic, University Malaysia.

[16] J. Fierrez-A , 2006, "Adapted Fusion Schemes for Multimodal Biometric Authentication" Ph.D thesis, Universidad Politecnica de Madrid.

[17] Vielhauer C. , 2005, “ Biometric User Authentication for It Security: From IT Security “ e. book, Published: New York : Springer.

[18] Karthik N. ,2005, " Integration of Multiple Cues in Biometric Systems “ Ph.D thesis Submitted to Michigan State University in partial fulfillment of the requirements for the degree of Master of Science CDepartment of Computer Science and Engineering.

[19] Bhanu B. , 2011, "Multibiometrics for Human Identification" Edited by University of California, Riverside Venu Govindaraju State University of New York, Buffalo.

[20] Boulgouris N. V. et al,2008, “ Theory, Methods, and Applications “ IEEE Computational Intelligence Society, Sponsor IEEE Press Series on Computational Intelligence.

[21] Kryszczuk K. and Drygajlo A. , 2009, "Improving Biometric Verification with Class-Independence quality information " Swiss Federal Institute of Technology Lausanne (EPFL).

[22] Nageshkumar M. ,2009 “ An Efficient Secure Multimodal Biometric Fusion Using Palmprint and Face Image" IJCSI International Journal of Computer Science Issues, Vol. 2.

[23] Sanghamitra B. , 2008, "Winter School on Data Mining and Computational Biology", of Indian Statistical Institute (ISI), Manipur University, Canchipur, Imphal.

[24] Wayman James, 2011 "Introduction to Biometrics " book in Library of Congress Control Number: 942231.

[25] Chen and A.K. Jain., 2005;"Dental biometrics: alignment and matching of dental radiographs. Pattern Analysis and Machine Intelligence", IEEE Transactions on, 27(8):13191326.

[26] Chaunssot J. , 2004, ” Filter with Progressively Decimated Filtering Windiw: Application To Color ,Image Enhancement “ Lis/ENSIEG-BP 46 IEEE vol7803-8554.

[27] Herman, G.T., 2009," Fundamentals of Computerized Tomography" Image Reconstruction from Projections" 2nd edu. Springer,Heidelberg.

[28] Lee, B.,2010, "Comparative study of attribute selection using gain ratio and correlation based feature selection," International Journal of Information Technology and Knowledge Management, pp. 271-277.

[29] Jie Zhu et al. 2011 "Implementation of Modified KL Transform for LPR Systems" European Journal of Scientific Research ISSN 1450-216X Vol.67 No.4 (2012), pp. 521-531, Euro Journals Publishing, Inc.

[30] Nagaveni. N.,"2011, "Semantically Enhanced Document Clustering Based on PSO Algorithm" book European Journal of Scientific Research ISSN 1450-216X Vol.57 No.3, pp.485-493, EuroJournals Publishing, Inc.

[31] Sivaskar E.,2011, "Comparison of Dimensionality Reduction Education Techniques Using a Backpropagation Neural Network Based Classifier “ Ms.H Department of Computer Science and Engineering National Institute of Technology Tiruchirappalli Tamil Nadu, India 